\title{
Correction to: Re-challenge of afatinib after 1st generation EGFR-TKI failure in patients with previously treated non-small cell lung cancer harboring EGFR mutation
}

\author{
Ou Yamaguchi ${ }^{1} \cdot$ Kyoichi Kaira $^{1}$ - Atsuto Mouri ${ }^{1}$. Ayako Shiono ${ }^{1} \cdot$ Kosuke Hashimoto $^{1} \cdot$ Yu Miura $^{1}$. \\ Fuyumi Nishihara ${ }^{1} \cdot$ Yoshitake Murayama $^{1} \cdot$ Kunihiko Kobayashi $^{1} \cdot$ Hiroshi Kagamu $^{1}$
}

Published online: 8 November 2019

(c) Springer-Verlag GmbH Germany, part of Springer Nature 2019

\section{Correction to: \\ Cancer Chemotherapy and Pharmacology (2019) 83:817-825 \\ https://doi.org/10.1007/s00280-019-03790-w}

In the original publication of the article, the authors found few errors and the corrections are given below:

\section{Abstract}

Results: "By multivariate analysis, smoking, performance status (PS), and time on treatment of 1st TKI with more than 10 months were confirmed to be independent prognostic factors predicting a worse progression-free survival" has been changed to "By multivariate analysis, smoking, performance status (PS) and time on treatment of 1st TKI with more than 10 months were confirmed to be independent prognostic factors predicting a better progressionfree survival (PFS)".

\section{Results}

\section{Patient demographics}

"EGFR mutation analysis revealed 35 patients with deletion of exon19, 19 patients with L858R (exon 21), and 2 patients with other mutations" has been changed to "EGFR mutation analysis revealed 35 patients with deletion of exon

The original article can be found online at https://doi.org/10.1007/ s00280-019-03790-w.

\section{Kyoichi Kaira}

kkaira1970@yahoo.co.jp

1 Department of Respiratory Medicine Comprehensive Cancer Center, International Medical Center, Saitama Medical University, 1397-1 Yamane, Hidaka-City, Saitama 350-1298, Japan
19, 19 patients with L858R (exon 21), and 8 patients with other mutations".

3. In the online publication of the article, the values in the Table 2 mentioned under the column smoking $(N=62)$ and also value under the Gender, the column under the PFS (HR), has incorrectly published. The corrected Table 2 has been given below:

4. Under the figure 1, the text in the figure legends has been incorrectly published. The corrected text is given below:

"A statistically significant difference in the PFS $(p=0.22)$ (C) and OS $(p=0.44)$ (D) was not observed in the patients between more than and less than 6 months".

5. The captions for the Figure 2 has been changed from

a) TTF of prior EGFR-TKI: $>10$ months $\leq 10$ months to Time on treatment of prior EGFRTKI: $>10$ months $\leq 10$ months

b) TTF of prior EGFR-TKI: $>10$ months $\leq 10$ months to Time on treatment of prior EGFR-TKI: $>10$ months $\leq 10$ months.

The corrected Fig. 2 has been given below:

6. In the online published article, the Figure legends under the figure 2 has been changed from

"Forest plot of 5-year PFS (a) and OS (b) rates according to time on treatment using cut-off values of 10 months in each variables" to

"Forest plot of PFS (a) and OS (b) rates according to time on treatment using cut-off values of 10 months in each variables". 
Table 2 Univariate survival analysis according to different variables

\begin{tabular}{|c|c|c|c|c|c|c|c|c|c|}
\hline \multirow[t]{2}{*}{ Variables } & \multirow[t]{2}{*}{$N=62$} & \multicolumn{4}{|l|}{ PFS } & \multicolumn{4}{|l|}{ OS } \\
\hline & & Median (days) & HR & $95 \% \mathrm{CI}$ & $p$ value & Median (days) & HR & $95 \% \mathrm{CI}$ & $p$ value \\
\hline \multicolumn{10}{|l|}{ Age } \\
\hline$\leq 69 />69$ years & $35 / 27$ & $174 / 194$ & 1.12 & $0.64-1.92$ & 0.15 & $572 / 485$ & 1.42 & $0.76-2.65$ & 0.26 \\
\hline \multicolumn{10}{|l|}{ Gender } \\
\hline Male/female & $25 / 37$ & $144 / 209$ & 1.36 & $0.91-3.03$ & 0.09 & $532 / 587$ & 0.96 & $0.51-1.81$ & 0.92 \\
\hline \multicolumn{10}{|l|}{ Smoking } \\
\hline Yes/no & $28 / 34$ & $213 / 94$ & 0.44 & $0.24-0.82$ & $<0.01$ & $572 / 551$ & 0.91 & $0.49-1.68$ & 0.75 \\
\hline \multicolumn{10}{|l|}{ ECOG PS } \\
\hline $0-1 / 2-3$ & $42 / 20$ & $227 / 73$ & 0.43 & $0.23-0.83$ & 0.01 & $778 / 153$ & 0.32 & $0.15-0.67$ & $<0.01$ \\
\hline \multicolumn{10}{|l|}{ BSA } \\
\hline$\leq 1.5 />1.5$ & $43 / 19$ & $168 / 227$ & 0.77 & $0.43-1.39$ & 0.39 & $501 / 904$ & 0.74 & $0.38-1.45$ & 0.39 \\
\hline \multicolumn{10}{|l|}{ EGFR mutation status } \\
\hline Del 19/L858R & $35 / 19$ & $174 / 168$ & 0.99 & $0.54-1.81$ & 0.98 & $754 / 505$ & 0.59 & $0.28-1.24$ & 0.12 \\
\hline \multicolumn{10}{|c|}{ Time on treatment of prior EGFR-TKI (months) } \\
\hline$\leq 6 />6$ & $7 / 55$ & $60 / 195$ & 0.19 & $0.05-0.66$ & $<0.01$ & $145 / 587$ & 0.21 & $0.05-0.78$ & $\mathbf{0 . 0 2}$ \\
\hline$\leq 8 />8$ & $11 / 51$ & $94 / 195$ & 0.33 & $0.13-0.84$ & 0.02 & $389 / 587$ & 0.32 & $0.11-0.98$ & 0.04 \\
\hline$\leq 10 />10$ & $16 / 46$ & $73 / 222$ & 0.41 & $0.19-0.87$ & 0.02 & $389 / 587$ & 0.36 & $0.14-0.87$ & 0.02 \\
\hline$\leq 12 />12$ & $17 / 45$ & $85 / 209$ & 0.43 & $0.21-0.91$ & 0.02 & $389 / 587$ & 0.42 & $0.18-0.96$ & 0.04 \\
\hline$\leq 14 />14$ & $22 / 40$ & $73 / 238$ & 0.36 & $0.18-0.71$ & $<0.01$ & $389 / 667$ & 0.34 & $0.16-0.72$ & $<0.01$ \\
\hline$\leq 20 />20$ & $32 / 30$ & $109 / 256$ & 0.45 & $0.25-0.81$ & $<0.01$ & $532 / 754$ & 0.63 & $0.34-1.17$ & 0.14 \\
\hline \multicolumn{10}{|c|}{ Time from prior EGFR-TKI to afatinib (months) } \\
\hline$\leq 4 />4$ & $39 / 23$ & $168 / 209$ & 0.63 & $0.36-1.11$ & 0.11 & $505 / 704$ & 0.68 & $0.36-1.26$ & 0.22 \\
\hline$\leq 6 />6$ & $42 / 20$ & $194 / 191$ & 0.71 & $0.40-1.24$ & 0.22 & $532 / 704$ & 0.78 & $0.41-1.46$ & 0.44 \\
\hline$\leq 12 />12$ & $52 / 10$ & $194 / 134$ & 0.60 & $0.29-1.21$ & 0.15 & $551 / 704$ & 0.82 & $0.37-1.76$ & 0.61 \\
\hline \multicolumn{10}{|c|}{ Cytotoxic agent between prior EGFR-TKI and afatinib } \\
\hline Yes/no & $31 / 31$ & $194 / 164$ & 0.73 & $0.42-1.27$ & 0.26 & $532 / 704$ & 0.53 & $0.28-1.00$ & 0.05 \\
\hline \multicolumn{10}{|c|}{ Initial dosage of afatinib } \\
\hline $40 / 20$ or $30 \mathrm{mg}$ & $46 / 16$ & $171 / 238$ & 1.22 & $0.67-2.21$ & 0.52 & $551 / 667$ & 1.21 & $0.59-2.43$ & 0.61 \\
\hline \multicolumn{10}{|c|}{ Disease stage at the initiation of prior EGFR-TKI } \\
\hline $\begin{array}{l}\text { IV/recurrence after } \\
\text { surgical resection }\end{array}$ & $43 / 19$ & $174 / 194$ & 1.46 & $0.83-2.03$ & 0.19 & $551 / 587$ & 1.42 & $0.76-2.67$ & 0.29 \\
\hline \multicolumn{10}{|c|}{ Treatment line of afatinib } \\
\hline$\leq 3 />3$ lines & $33 / 29$ & $213 / 168$ & 0.66 & $0.38-1.13$ & 0.11 & $572 / 551$ & 1.02 & $0.55-1.86$ & 0.95 \\
\hline \multicolumn{10}{|c|}{ Numbers of initial EGFR-TKI before afatinib } \\
\hline $1 / 2$ & $36 / 26$ & $209 / 156$ & 0.59 & $0.34-1.03$ & 0.05 & $667 / 437$ & 0.59 & $0.32-1.09$ & 0.08 \\
\hline
\end{tabular}

Bold indicates statistically significant difference

ECOG eastern cooperative oncology group, $P S$ performance status, $P F S$ progression free survival, $O S$ overall survival, $H R$ hazard ratio, $95 \% C I$ 95\% confidence interval, EGFR epidermal growth factor receptor, $T K I$ tyrosine kinase inhibitor, $B S A$ body surface area 


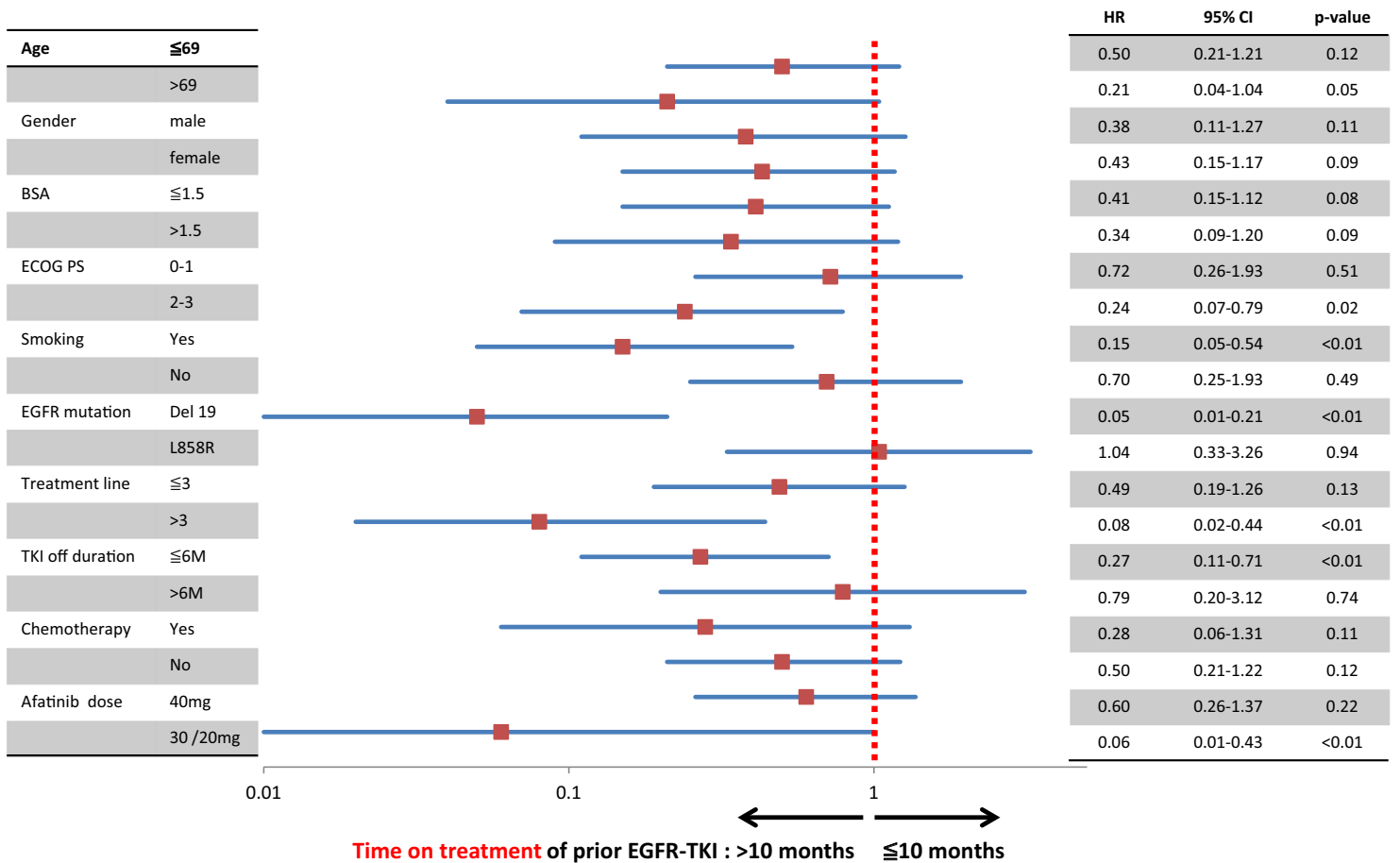

Fig. 2 Series 62 Model 4666 extrapolations

Publisher's Note Springer Nature remains neutral with regard to jurisdictional claims in published maps and institutional affiliations. 